Service social

\title{
Psychologie du couple : quand la science se met à parler d'amour, par Madeleine Beaudry et Jean-Marie Boisvert, Montréal, Éditions du Méridien, 1988, 405 pages.
}

\section{Germain Trottier}

Volume 38, numéro 1, 1989

Aspects psychosociaux du Sida

URI : https://id.erudit.org/iderudit/706431ar

DOI : https://doi.org/10.7202/706431ar

Aller au sommaire du numéro

Éditeur(s)

École de service social de l'Université Laval

ISSN

1708-1734 (numérique)

Découvrir la revue

Citer ce compte rendu

Trottier, G. (1989). Compte rendu de [Psychologie du couple : quand la science se met à parler d'amour, par Madeleine Beaudry et Jean-Marie Boisvert, Montréal, Éditions du Méridien, 1988, 405 pages.] Service social, 38(1), 138-140.

https://doi.org/10.7202/706431ar d'utilisation que vous pouvez consulter en ligne.

https://apropos.erudit.org/fr/usagers/politique-dutilisation/ 
population hétérosexuelle. II apparaît alors une "exploitation politique du problème" (p. 19). Pour Pollak, ce débat se poursuivra : "les réactions évolueront probablement de façon différentielle, en fonction essentiellement de la représentation du risque et du sentiment individuel ou collectif de pouvoir s'adapter ou non. Le savoir et les croyances sur la contamination interviennent autant dans ces réactions que les angoisses projetées sur le risque biologique" (p. 199-200).

En définitive, vis-à-vis sa question de départ portant sur la prévention, l'auteur ne fournit pas une réponse unique. Dans le respect des droits de chacun, il privilégie, particulièrement auprès de clientèles ciblées, des démarches de promotion de gestes préventifs, tels le test volontaire de dépistage et le support à la responsabilisation individuelle. Nous déplorons toutefois que le dernier chapitre et la conclusion prospective ne répondent que de façon parcellaire à l'interrogation initiale. Pourtant, la description qu'il fournit de la gestion du risque par la communauté gaie française, de la crise d'identité chez les homosexuels interviewés et de la modification des pratiques sexuelles, suscite néanmoins des pistes de réflexion pour l'élaboration de démarches préventives. Ce champ d'intervention reste donc ouvert à des investigations complémentaires.

Par ailleurs, même si ces comptes rendus de recherche et cette discussion des rapports de forces informent sur la situation sociale et politique en France eu égard au sida, ils peuvent soutenir nos propres questions en contexte québécois et canadien. Quel est par exemple notre niveau de tolérance et d'acceptation? Pour terminer, nous aimerions souligner la pertinence de l'utilisation des données qualitatives d'entrevue en alternance avec les résultats statistiques, afin de saisir l'intensité des réactions provoquées par le sida.

Marie Drolet

Étudiante au doctorat en service social,

Université Laval.

Psychologie du couple : quand la science se met à parler d'amour, par Madeleine BeaUdRY et Jean-Marie BoIsvert, Montréal, Éditions du Méridien, 1988, 405 pages.

Au premier abord, l'ouvrage pique la curiosité par l'énoncé global de son titre et de son graphisme : $y$ chercher un lien possible vous ramène à tort ou à raison à l'interprétation libre, le meilleur choix. Mais comme il se doit, la préface nous renseigne sur le type de lecteurs-cibles à qui ce volume est destiné ; avant tout, aux professionnels et futurs professionnels de la thérapie de couple mais également à tout individu, homme et femme, que la vie amoureuse comble ou qui se sent malheureux dans la vie à deux. En somme, une sorte de traité où chacun trouve son profit.

Le volume de 405 pages, composé de douze chapitres sensiblement égaux, réussit à faire l'étude de la vie amoureuse des humains à la lumière d'une approche rigoureuse et systématique. On y traite tour à tour des aspects 
suivants : les théories explicatives sur la relation de couple, la recension des principaux écrits sur le sujet, la dynamique des relations amoureuses et ses enjeux, les méthodes d'évaluation utilisables, les modes d'intervention stratégiques, les mises en garde appropriées pour une action efficace. L'appendice du volume comprend un ensemble d'exercices simples et utiles à l'intention du lecteur. Les références sont précises et abondantes et, fait à noter, un index des sujets abordés s'y ajoute pour une utilisation fonctionnelle de l'ouvrage. En somme, comme l'annoncent les auteurs, il s'agit d'un manuel d'introduction à la thérapie conjugale.

Au fond, Beaudry et Boisvert rendent un grand service à la société en publiant un tel volume. D'une part, ils abordent dans des propos simples et concrets ce qui constitue la trame essentielle de la vie humaine, à savoir le besoin d'aimer et d'être aimé, mais en même temps, ils réussissent à traduire combien l'aventure est difficile et périlleuse pour qui n'est pas d'abord à l'écoute de son individualité. D'autre part, ils enseignent aux professionnels de l'aide comment guider avec doigté des personnes dans leur recherche du bonheur à deux. Comme deux penseurs expérimentés, ils proposent des pistes théoriques et pratiques qui ne s'éloignent jamais de l'approche scientifique recherchée au départ. Leur influence en milieu québécois sera certes de notoriété.

Ceci dit pour l'ensemble, nous estimons toutefois qu'un chapitre de l'ouvrage (chap. 10) présente quelques faiblesses. À notre avis, la démarche de résolution de problèmes ne se limite pas à identifier les difficultés à résoudre les problèmes et à imaginer des solutions, mais également à reconnaître ce qui a pu être expérimenté avec succès, dans le passé comme dans le présent, afin de tirer parti au maximum des expériences positives relevées. Les auteurs ne mettent pas suffisamment l'accent sur ce point, ce qui, à mon avis, diminue l'importante nécessité d'entretenir chez les gens concernés l'idée qu'ils peuvent solutionner eux-mêmes leurs problèmes. Cette lacune contribue bien malheureusement à entretenir la pensée magique que les professionnels sont là pour porter les problèmes des gens qui les consultent. Nous n'insisterons jamais assez pour démanteler les schèmes qui font des professionnels de l'aide les sauveurs uniques de "situations problèmes".

Pour boucler le processus scientifique amorcé, il aurait fallu souligner davantage l'importance d'une évaluation partagée des résultats de l'intervention. Outre les questions d'éthique abordées, il nous aurait paru essentiel d'insister sur le partage des progrès accomplis avec l'aide du professionnel pour dégager ce que les personnes peuvent poursuivre d'elles-mêmes. Tel quel, les auteurs paraissent avoir manqué d'un peu de souffle pour mener à bien les objectifs qu'ils tentaient d'atteindre.

En dépit de ces quelques faiblesses, l'ouvrage de Beaudry et Boisvert constitue un tribut remarquable aux professionnels de la thérapie conjugale et à leurs aspirants. Leur contribution à l'avancement des connaissances sur la vie à deux sera notable pour l'avenir et une source à utiliser en formation.

Pour terminer, on pourrait attribuer à Beaudry et Boisvert cette idée que le spécialiste du bonheur "cherche le commun dénominateur des bonheurs 
particuliers pour ensuite définir les aptitudes qu'il convient de développer pour le rendre aisément accessible et, même, pour le généraliser " ${ }^{1}$

Germain Trottier

École de service social,

Université Laval.

\section{Notes et références}

* Le masculin est utilisé sans discrimination et dans le seul but d'alléger le texte.

1 BLONDIN, René, Le bonheur possible, Montréal, Les Éditions de l'homme, 1983, p. 11.

Guide d'intervention de groupe auprès des parents d'adolescents, par Michel Y. Ménard et Camile SPAIN, Pierrefonds, C.L.S.C. Pierrefonds, 1989, 84 pages.

D'une façon détaillée, ce guide expose le programme Parents d'adolescents bien branchés mis en place au C.L.S.C. Pierrefonds depuis 1981. Ce programme a pour but de "permettre aux parents d'adolescents d'accroître leur compétence parentale pour améliorer les relations parents/adolescents» (p. 12). Il en découle des objectifs aux niveaux cognitif, affectif et comportemental, par exemple améliorer ses connaissances sur l'adolescence, développer une prise de conscience de ses attitudes ou maîtriser une méthode de résolution des conflits.

Mettant en évidence les besoins des parents d'adolescents dans leur tâche d'éducation, les auteurs souhaitent que les personnes qui veulent travailler en groupe auprès de cette population trouvent dans cet ouvrage des idées, des exercices et même une méthode globale d'animation d'une rencontre thématique.

Quittant, tel qu'il est mentionné dans la préface, le "sentier officiel des méthodes à idéologie unique ", les auteurs ont trouvé leurs sources d'inspiration dans des expériences faites au Bureau de Consultation-Jeunesse, à la Commission des Écoles Catholiques de Montréal et au C.L.S.C. Centre-Sud. Au plan théorique, ils se réfèrent surtout au service social des groupes (groupe structuré d'éducation et modèle du courant central).

Après une présentation des activités de la phase pré-groupe, le guide se divise en dix parties correspondant aux thèmes de chacune des rencontres: le contrat, la psychologie de l'adolescence, l'initiation à la communication, l'écoute empathique, l'expression des sentiments, les drogues et la rivalité fraternelle, la sexualité, la résolution des conflits, la motivation scolaire et les loisirs, ainsi que l'honnêteté et l'évaluation des acquis. On retrouve aussi, pour chacune de ces rencontres, une procédure identique: une présentation du contenu de la rencontre, un exercice de réchauffement, un retour sur l'exercice à la maison, 